\title{
Magnetic resonance image-based tomotherapy planning for prostate cancer
}

\author{
Sang Hoon Jung ${ }^{1 *}$, Jinsung Kim", Yoonsun Chung ${ }^{3}$, Bilgin Keserci ${ }^{4,5}$, Hongryull Pyo ${ }^{6}$, Hee Chul Park ${ }^{6}$, Won Park \\ ${ }^{1}$ Department of Radiation Oncology, Samsung Medical Center, Seoul, Korea \\ ${ }^{2}$ Department of Radiation Oncology, Yonsei Cancer Center, Yonsei University College of Medicine, Seoul, Korea \\ ${ }^{3}$ Department of Nuclear Engineering, Hanyang University, Seoul, Korea \\ ${ }^{4}$ Department of Radiology, School of Medical Sciences, Universiti Sains Malaysia, Kelantan, Malaysia \\ ${ }^{5}$ Department of Radiology, Hospital Universiti Sains Malaysia (USM), Kelantan, Malaysia \\ ${ }^{6}$ Department of Radiation Oncology, Samsung Medical Center, Sungkyunkwan University School of Medicine, Seoul, Korea
}

Received: February 28, 2020

Revised: March 19, 2020

Accepted: March 20, 2020

\section{Correspondence:}

Won Park

Department of Radiation Oncology,

Samsung Medical Center,

Sungkyunkwan University School of

Medicine, 81 Irwon-ro, Gangnam-gu,

Seoul 06351, Korea

Tel: +82-2-3410-2616

Fax: +82-2-3410-3619

E-mail:wonro.park@samsung.com

ORCID:

https://orcid.org/0000-0003-4686-2071

*These authors contributed equally to this work.
Purpose: To evaluate and compare the feasibilities of magnetic resonance (MR) image-based planning using synthetic computed tomography (sCT) versus $\mathrm{CT}$ ( $\mathrm{pCT}$ )-based planning in helical tomotherapy for prostate cancer.

Materials and Methods: A retrospective evaluation was performed in 16 patients with prostate cancer who had been treated with helical tomotherapy. MR images were acquired using a dedicated therapy sequence; sCT images were generated using magnetic resonance for calculating attenuation (MRCAT). The three-dimensional dose distribution according to SCT was recalculated using a previously optimized plan and was compared with the doses calculated using pCT.

Results: The mean planning target volume doses calculated by SCT and pCT differed by $0.65 \% \pm$ $1.11 \%(p=0.03)$. Three-dimensional gamma analysis at a $2 \% / 2 \mathrm{~mm}$ dose difference/distance to agreement yielded a pass rate of 0.976 (range, 0.658 to 0.986 ).

Conclusion: The dose distribution results obtained using tomotherapy from MR-only simulations were in good agreement with the dose distribution results from simulation CT, with mean dose differences of less than 1\% for target volume and normal organs in patients with prostate cancer.

Keywords: Prostatic neoplasms, Magnetic resonance imaging, Helical tomotherapy

\section{Introduction}

Image-guided intensity-modulated radiotherapy (IG-IMRT) has been widely used in the treatment of prostate cancer, with the goal of reducing acute and late toxicity of adjunct critical organs, e.g., the bladder and rectum [1,2]. Helical tomotherapy is considered a favorable treatment technique for prostate cancer due to its uniformity in treatment and the fact that it is based on computed tomography $(\mathrm{CT})$ simulation $[3,4]$.

Magnetic resonance imaging (MRI) has been shown to be supe- rior to CT with respect to the characterization and visualization of soft tissue. Compared to CT with target delineation, CT-MR fusion images enable more precise identification of the target volume in the prostate, seminal vesicles, and pelvic lymphatics [5-8] and thus have the potential to reduce complications of critical organs $[9,10]$.

However, the uncertainty of CT-MR registration can propagate to target delineation [11]. To eliminate this uncertainty, MR-only simulations in radiotherapy (RT) can be used [12]. Various techniques for creating synthetic CT (sCT) images have been proposed and validated, e.g., assigning fixed densities to tissues [13], using

Copyright@ 2020 The Korean Society for Radiation Oncology

This is an Open Access article distributed under the terms of the Creative Commons Attribution Non-Commercial License (http://creativecommons.org/licenses/by-nc/4.0/) which permits unrestricted non-commercial use, distribution, and reproduction in any medium, provided the original work is properly cited. 
atlas-based segmentation from databases $[14,15]$, and correlating Hounsfield units (HUs) and MR intensities [16,17].

The aim of this study was to evaluate a technique for generating SCT images using a dedicated MRI pulse sequence and an algorithm in a dedicated MR-RT simulator. Additionally, we aimed to calculate the residual treatment dose discrepancy between planning CT (pCT) and sCT using a helical tomotherapy plan for prostate cancer as a reference. Finally, we set out to assess the feasibility of MR-only simulations with helical tomotherapy for patients with prostate cancer.

\section{Materials and Methods}

\section{Patient characteristics}

This study included treatment plans for 16 patients with prostate cancer who were treated with helical tomotherapy. The patient characteristics are summarized in Table 1. The median patient age was 73 years (range, 59 to 83 years). Eleven patients were treated with definitive (curative intent) RT, while the rest were treated with salvage RT. Surgical bed was included in the treatment field for 4 patients; elective pelvic lymph node areas were additionally treated in 12 patients. This study was approved by the Institutional Review Board of Samsung Medical Center, Korea approved this

Table 1. Patients' characteristics $(n=16)$

\begin{tabular}{lc}
\hline Characteristic & Value \\
\hline Age $(\mathrm{yr})$ & $73(59-83)$ \\
T stage & $3(18.75)$ \\
T2 & $5(31.25)$ \\
T3a & $7(43.75)$ \\
T3b & $1(6.25)$ \\
T4 & \\
Initial PSA (ng/mL) & $4(25.00)$ \\
$<10$ & $4(25.00)$ \\
10-20 & $8(60.00)$ \\
$>20$ & \\
Grade & $2(12.50)$ \\
6 & $3(18.75)$ \\
7 & $11(68.75)$ \\
$8-9$ & \\
Treatment aim & $11(68.75)$ \\
Definitive & $5(31.25)$ \\
Salvage & \\
Radiation field & $4(25.00)$ \\
Surgical bed only & $12(75.00)$ \\
Whole pelvis &
\end{tabular}

Values are presented as median (range) or number (\%). PSA, prostate-specific antigen. retrospective study (No. 2016-07-109-001). The requirement for informed consent was waived.

\section{2. $\mathrm{pCT}$ imaging}

Each patient was immobilized using a vacuum cushion and positioned in the head-first supine position with both arms placed on the chest. A catheter with a small rectal balloon filled with $60 \mathrm{~mL}$ of air was inserted into the rectum. Three-dimensional (3D) CT images (pixel spacing $0.98 \mathrm{~mm} \times 0.98 \mathrm{~mm}, 512 \times 512$ ) were acquired using a Discovery CT590RT (GE Healthcare, Waukesha, WI, USA). The slice thickness was $2.5 \mathrm{~mm}$. Contrast medium (90 mL) was injected at a flow rate of $2 \mathrm{~mL} / \mathrm{s}$.

\section{MR imaging and generation of sCT images}

MR scans were conducted immediately after the PCT scans on the same day using a 3.0 T Ingenia MR simulation system (Philips Healthcare, Best, The Netherlands). These systems are dedicated for radiation therapy and are equipped with a 16-channel abdominal receiving coil and integrated posterior coil assembly. The patient setup was the same as in the pCT simulation, with the exception that the feet-first-supine orientation was used. This position allowed more flexibility than the head-first setup when positioning the anterior receiving coil. A dedicated $3 \mathrm{D}$ radiofrequency spoiled incoherent gradient 2-echo instrument (3D-T1-FFE 2-echo; repetition time [TR], $3.8 \mathrm{~ms}$; echo time-1 [TE1], $1.2 \mathrm{~ms}$; TE2, $2.4 \mathrm{~ms}$; flip angle, $10^{\circ}$ ) was used to collect the source images. Two sets of T1-weighted MR images with different TEs were obtained.

Next, two-point mDixon reconstruction was used to generate three sets of MR images: in-phase, water-only, and fat-only (Fig. 1A, $1 \mathrm{~B}$, and $1 \mathrm{C}$, respectively). To generate the $\mathrm{sCT}$ images, a dedicated post-processing algorithm $[18,19]$ was utilized. The sCT images were generated automatically using a magnetic resonance calculating attenuation (MRCAT) algorithm, as shown in Fig. 1D. The MRCAT sCT images consisted of air, fat, normal tissue, spongy bone, and compact bone; the materials were assigned -968, -86, 42, 198, and 949 HUs, respectively. The sCT images were $432 \times$ 432 pixels, with pixel spacing of $1.04 \mathrm{~mm} \times 1.04 \mathrm{~mm}$ and a slice thickness of $2.5 \mathrm{~mm}$.

\section{Treatment plans for tomotherapy on $\mathrm{pCT}$}

In treatment planning, 3D CT images were used as the reference (pCT). The primary clinical target volume (CTV1), which included the surgical bed, was delineated on the PCT images using a Pinnacle $^{3}$ system (v.9.10; Philips Medical Systems, Fitchburg, WI, USA). Pelvic lymphatics were delineated as the secondary clinical target volume (CTV2) in 12 patients. A setup margin (SM) of 3-5 mm was added to the CTV1 to create the planning target volume (PTV1), 

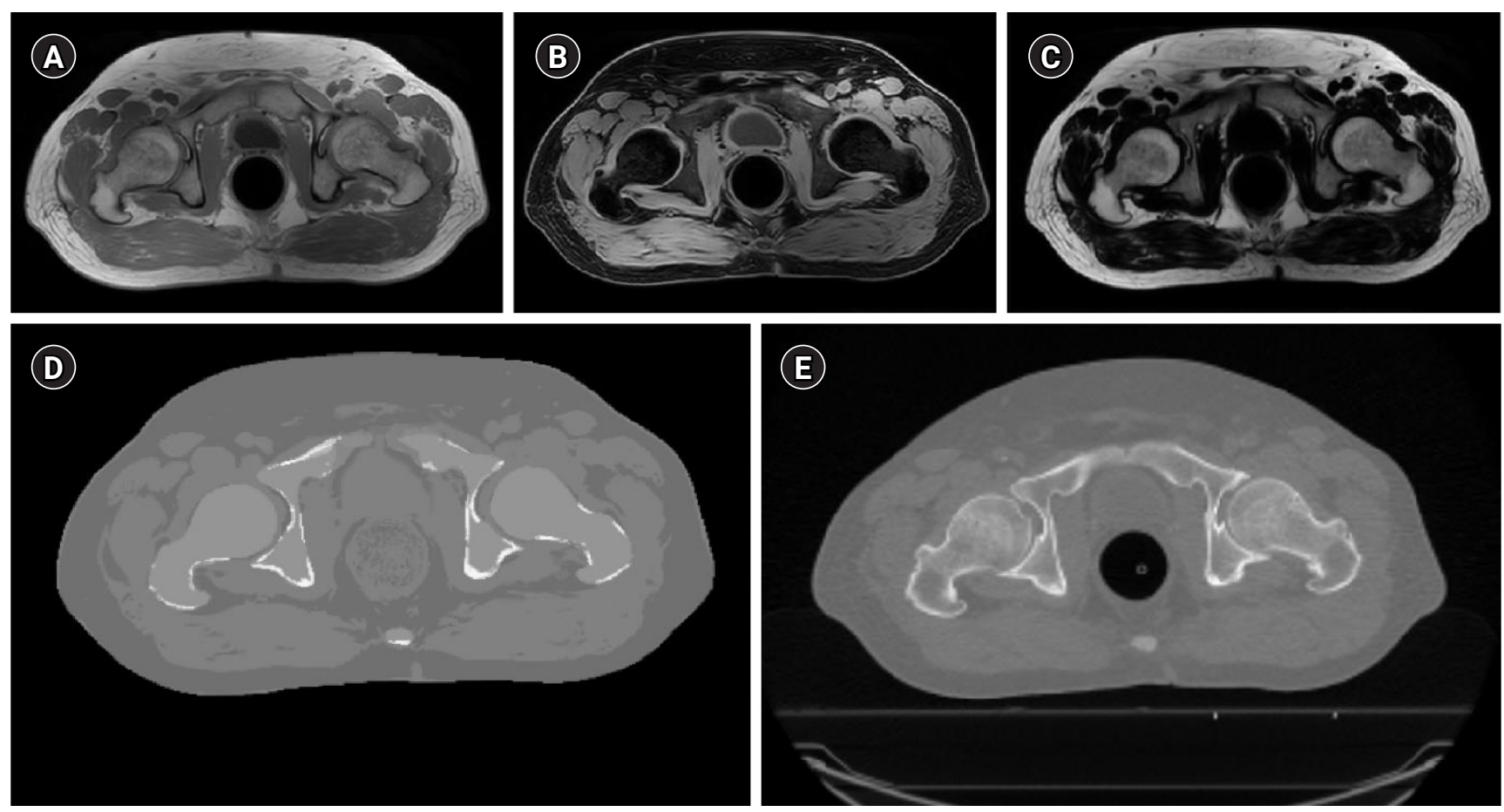

Fig. 1. Representative magnetic resonance image used for calculation attenuation (MRCAT). (A) In-phase, (B) water-only, and (C) fat-only MR images for patient \#13 were generated from the three-dimensional T1-weighted mDixon images using 2 echoes. (D) The synthetic computed tomography (CT) image was generated using the MRCAT and MR images. (E) A planning CT image was acquired from the CT simulation.

and an SM of 5-7 mm was added to the CTV2 to create the PTV2.

IMRT plans were performed using TomoTherapy planning stations (Hi-Art ver. 4.2.3 or TomoHD ver. 1.2.3; Accuray Inc., Sunnyvale, $C A$, USA). The prescribed doses to the PTV1 $\left(1^{\circ} D_{\text {pre }}\right)$ and PTV2 $\left(2^{\circ} \mathrm{D}_{\text {pre }}\right)$ were $70.0 \mathrm{~Gy}$ and $50.4 \mathrm{~Gy}$, respectively, at 28 fractions. The dose constraints in the treatment plans were: (1) the $1^{\circ} D_{\text {pre }}$ had to cover at least $98 \%$ of the PTV, and (2) the maximum dose $\left(D_{\max }\right)$ of the PTV had to be less than $107 \%$ of the $1{ }^{\circ} D_{\text {pre. }}$ Doses to organsat-risk (OARs), such as the rectum wall, bladder, penile bulb, femoral heads, and bowel, were also considered.

\section{Dose calculation on sCT}

The 3D dose distribution based on the SCT results was calculated using the optimized reference treatment plan on the pCT images. To this end, the delivery quality application (DOA) of a tomotherapy workstation was used. The SCT image was registered rigidly to the pCT image and resampled to match the dimensions and patient positions of the SCT image to those of the pCT image using MIM Maestro ver. 6.4.9 (MIM Software, Cleveland, OH, USA). The structures of the target volumes and normal organs delineated on pCT were copied to the structures on SCT. All registrations and structures on SCT were validated by both a physician and a physicist.

The couchtop was not visible on the sCT image from the MRI scan. Since the sCT image was already registered to the pCT image, a virtual couch was inserted into the SCT image at the same position of the pCT image in the DICOM coordination. The sCT images were modified to be similar to phantom images [20] and were then imported into the DQA workstation for recalculation of the 3D dose.

\section{Dosimetric analysis}

To evaluate the clinical feasibility of using SCT in a tomotherapy-based treatment plan, the image values, dosimetric parameters, and $3 \mathrm{D}$ doses recalculated using the $\mathrm{SCT}$ images were compared to those calculated using the pCT images. Specifically, the mean HUs of the target volumes, OARs, and materials on the pCT and SCT images were calculated and compared. Dosimetric parameters such as $D_{\text {mean }} D_{5 \%}$, and $D_{95 \%}$ were calculated from the dose-volume histograms (DVHs) and compared in order to evaluate differences in the planned doses delivered to the target volumes; $D_{\text {mean }}$ and $D_{\max }$ were compared to evaluate dose differences to OARs. In addition, the 3D gamma values were evaluated with the global dose criteria of $3 \% / 3 \mathrm{~mm}-1 \% / 1 \mathrm{~mm}$ (dose difference/distance to agreement).

Statistical differences in the dosimetric parameters from the different dose distributions were assessed using the Wilcoxon 
signed-rank test. The test was performed using SPSS v. 20.0 (IBM Corp., Armonk, NY, USA). A p-value $\leq 0.05$ was considered to be statistically significant.

\section{Results}

\section{Differences in HUs}

After automatic generation of the SCT images from the MR images using the MRCAT algorithm, treatment plans were determined, and dose recalculations were performed using the SCT images. The differences in HUs between the sCT images and the pCT images are summarized in Table 2. The mean differences of the target volumes and OARs were evaluated to be less than 30, with the exception of the rectum wall and femoral heads. Since the air in the rectal balloon could not be represented on the SCT images by MRCAT, the greatest HU differences were observed for the rectum wall. The differences in the mean HUs for fat and muscle were evaluated to be less than 10 .

\section{Dosimetric parameters}

Three-dimensional dose for all the patients were re-calculated and DVHs were compared as shown in Fig. 2. Dosimetric parameters of the target volumes and OARs from the DVHs were summarized and compared in Table 3. The mean differences in $D_{\text {mean }} D_{95 \% \text {, }}$ and $D_{5 \%}$ for PTV1 and PTV2 between pCT and sCT were all less than $0.74 \mathrm{~Gy}$. The $D_{\text {mean }}$ and $D_{5 \%} s C T$ values were both statistically different from the PCT values; however, the absolute differences of the mean values of all parameters were less than 1.04\%. Due to the HU differ- ences for fat and muscle in the body outlines, the $D_{\text {mean }}$ and $D_{\max }$ values calculated from the SCT images for the rectum wall, right femoral head, left femoral head, and penile bulb were also significantly different from those calculated from the pCT images; in contrast, the bladder values were not significantly different. However, for all OARs, the normalized $D_{\text {mean }}$ and $D_{\max }$ values, using $1^{\circ} D_{\text {pre }}$ (70 Gy), were less than $0.59 \%$ and $1.59 \%$, respectively.

\section{Gamma evaluation}

The results of the 3D gamma analysis at $3 \% / 3 \mathrm{~mm}, 2 \% / 2 \mathrm{~mm}$, and $1 \% / 1 \mathrm{~mm}$ dose difference/distance to agreement are shown in Fig. 3. With respect to overall volume, gamma analysis revealed pass rates of 0.998 (range, 0.981 to 1.000 ), 0.976 (range, 0.658 to 0.986 ), and 0.871 (range, 0.540 to 0.931 ), with $\gamma_{50}$ values of 0.123 (range, 0.084 to 0.203 ), 0.190 (range, 0.128 to 0.460 ), and 0.379 (range, 0.257 to 0.920$)$, respectively. For the target volumes and critical organs, the median and mean pass rates at $3 \% / 3 \mathrm{~mm}$ and $2 \% / 2 \mathrm{~mm}$ were both greater than 0.95 , with $\gamma_{50}$ values less than 0.5 .

\section{Discussion and Conclusion}

We demonstrated the feasibility of MR-only simulations using helical tomotherapy for patients with prostate cancer. Tomotherapy and the associated planning algorithms comprise one of the most advanced radiation therapy techniques, while MR is the preferred medical imaging method for patients with prostate cancer. We successfully treated 16 patients with prostate cancer with conventional CT using tomotherapy and acquired MR images in the

Table 2. Comparison of mean image values (unit: HU)

\begin{tabular}{|c|c|c|c|c|}
\hline & Planning CT & Synthetic CT & Difference & p-value \\
\hline \multicolumn{5}{|l|}{ Target volumes } \\
\hline PTV1 $(n=16)$ & $14.0 \pm 24.9$ & $23.4 \pm 13.1$ & $9.5 \pm 26.1$ & 0.07 \\
\hline PTV2 (n = 12) & $33.4 \pm 14.2$ & $-6.8 \pm 8.0$ & $-30.2 \pm 20.3$ & $<0.01^{*}$ \\
\hline \multicolumn{5}{|c|}{ Normal organs $(n=16)$} \\
\hline Bladder & $18.6 \pm 7.1$ & $35.3 \pm 6.1$ & $16.7 \pm 10.5$ & $<0.01^{*}$ \\
\hline Rectum wall & $-143.3 \pm 156.6$ & $4.6 \pm 10.3$ & $147.9 \pm 154.4$ & $<0.01^{*}$ \\
\hline \multicolumn{5}{|l|}{ Femoral head } \\
\hline Right & $279.5 \pm 45.8$ & $175.5 \pm 6.1$ & $-104.0 \pm 47.5$ & $<0.01^{*}$ \\
\hline Left & $280.4 \pm 50.5$ & $177.7 \pm 6.4$ & $-102.7 \pm 51.7$ & $<0.01^{*}$ \\
\hline Penile bulb & $48.3 \pm 14.7$ & $40.3 \pm 2.5$ & $-8.0 \pm 14.0$ & $0.02^{*}$ \\
\hline \multicolumn{5}{|c|}{ Fat and muscle volumes $(n=16)$} \\
\hline Fat & $-69.4 \pm 13.6$ & $-77.3 \pm 1.4$ & $-7.9 \pm 13.0$ & $0.02^{*}$ \\
\hline Muscle & $34.1 \pm 12.4$ & $39.8 \pm 0.4$ & $5.7 \pm 12.3$ & $0.05^{*}$ \\
\hline
\end{tabular}

Values are presented as mean \pm standard deviation.

CT, computed tomography; HU, Hounsfield unit; PTV, planning target volume.

${ }^{*} p<0.05$, Wilcoxon paired signed-rank test. 

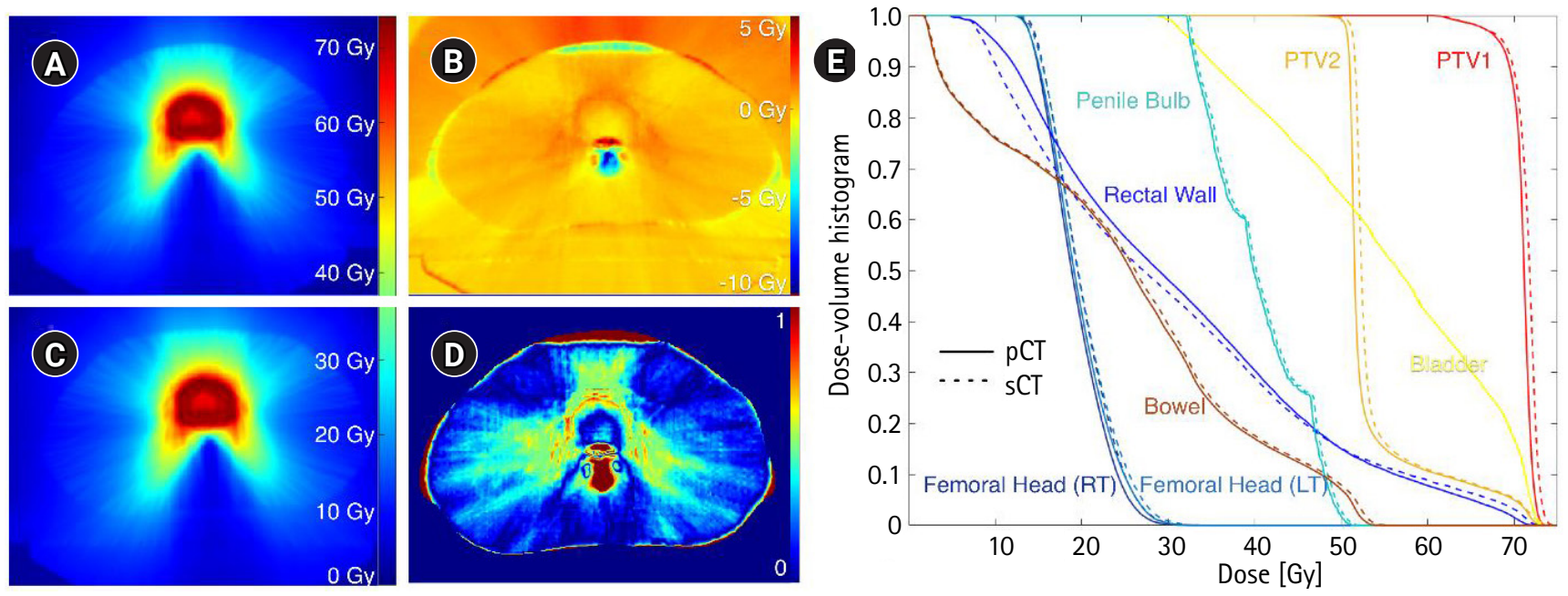

Fig. 2. Representative dosimetric results. $(A, B)$ display the three-dimensional dose distributions as calculated by planning computed tomography (CT) and synthetic CT images, respectively, for patient \#13. (C, D, E) present comparisons of the three-dimensional doses for patient \#13 with respect to dose difference, gamma analysis, and dose-volume histograms, respectively.

Table 3. Comparison of dosimetric parameters for target volumes, normal organs, and fat/muscle volumes

\begin{tabular}{|c|c|c|c|c|c|c|c|}
\hline Parameter & & & Planning CT (Gy) & Synthetic CT (Gy) & Difference (Gy) & $\begin{array}{c}\text { Normalized } \\
\text { difference (\%) }\end{array}$ & $p$-values \\
\hline \multirow[t]{6}{*}{ Target volumes } & \multirow[t]{3}{*}{ PTV1 $(n=16)$} & $\mathrm{D}_{\text {mean }}$ & $70.00 \pm 0.61$ & $70.46 \pm 1.07$ & $0.46 \pm 0.77$ & $0.65 \pm 1.11$ & $0.03^{*}$ \\
\hline & & $D_{95 \%}$ & $67.86 \pm 2.19$ & $68.20 \pm 2.17$ & $0.33 \pm 0.84$ & $0.48 \pm 1.19$ & 0.16 \\
\hline & & $D_{5 \%}$ & $71.47 \pm 0.79$ & $72.20 \pm 1.16$ & $0.73 \pm 0.74$ & $1.04 \pm 1.05$ & $<0.01^{*}$ \\
\hline & \multirow[t]{3}{*}{ PTV2 $(n=12)$} & $D_{\text {mean }}$ & $50.92 \pm 1.01$ & $51.24 \pm 1.21$ & $0.32 \pm 0.50$ & $0.34 \pm 0.65$ & 0.06 \\
\hline & & $D_{95 \%}$ & $47.17 \pm 2.44$ & $47.33 \pm 2.45$ & $0.12 \pm 0.48$ & $0.17 \pm 0.68$ & 0.34 \\
\hline & & $D_{5 \%}$ & $57.28 \pm 5.14$ & $57.75 \pm 5.24$ & $0.35 \pm 0.47$ & $0.50 \pm 0.67$ & $<0.01^{*}$ \\
\hline \multirow[t]{10}{*}{ Normal organs $(n=16)$} & \multirow[t]{2}{*}{ Bladder } & $D_{\text {mean }}$ & $40.19 \pm 7.19$ & $40.28 \pm 7.39$ & $0.09 \pm 0.52$ & $0.12 \pm 0.75$ & 0.98 \\
\hline & & $D_{\max }$ & $70.45 \pm 1.00$ & $70.55 \pm 1.34$ & $0.10 \pm 0.80$ & $0.14 \pm 1.14$ & 0.97 \\
\hline & \multirow[t]{2}{*}{ Rectum wall } & $D_{\text {mean }}$ & $30.72 \pm 3.06$ & $30.44 \pm 2.89$ & $-0.28 \pm 0.44$ & $-0.41 \pm 0.63$ & $0.03^{*}$ \\
\hline & & $D_{\max }$ & $70.02 \pm 1.10$ & $71.13 \pm 1.65$ & $1.11 \pm 0.86$ & $1.59 \pm 1.23$ & $<0.01^{*}$ \\
\hline & \multirow[t]{2}{*}{ Right femoral head } & $D_{\text {mean }}$ & $21.35 \pm 4.55$ & $21.68 \pm 4.64$ & $0.33 \pm 0.24$ & $0.48 \pm 0.34$ & $<0.01^{*}$ \\
\hline & & $D_{\max }$ & $34.92 \pm 7.72$ & $35.57 \pm 7.98$ & $0.65 \pm 0.43$ & $0.93 \pm 0.61$ & $<0.01^{*}$ \\
\hline & \multirow[t]{2}{*}{ Left femoral head } & $\mathrm{D}_{\text {mean }}$ & $20.93 \pm 4.10$ & $21.26 \pm 4.19$ & $0.33 \pm 0.23$ & $0.47 \pm 0.33$ & $<0.01^{*}$ \\
\hline & & $D_{\max }$ & $34.53 \pm 7.18$ & $35.21 \pm 7.48$ & $0.67 \pm 0.47$ & $0.96 \pm 0.67$ & $<0.01^{*}$ \\
\hline & \multirow[t]{2}{*}{ Penile bulb } & $D_{\text {mean }}$ & $39.05 \pm 14.86$ & $39.46 \pm 14.98$ & $0.41 \pm 0.41$ & $0.59 \pm 0.59$ & $<0.01^{*}$ \\
\hline & & $\mathrm{D}_{\max }$ & $52.42 \pm 14.84$ & $52.97 \pm 15.15$ & $0.55 \pm 0.69$ & $0.79 \pm 0.98$ & $<0.01^{*}$ \\
\hline \multirow[t]{4}{*}{ Fat and muscle volumes $(\mathrm{n}=16)$} & \multirow[t]{2}{*}{ Fat } & $D_{\text {mean }}$ & $11.16 \pm 4.00$ & $11.28 \pm 4.04$ & $0.12 \pm 0.08$ & $0.17 \pm 0.11$ & $<0.01^{*}$ \\
\hline & & $\mathrm{D}_{\max }$ & $46.65 \pm 9.14$ & $47.02 \pm 9.26$ & $0.37 \pm 0.51$ & $0.53 \pm 0.73$ & $0.01^{*}$ \\
\hline & \multirow[t]{2}{*}{ Muscle } & $D_{\text {mean }}$ & $12.66 \pm 4.15$ & $12.81 \pm 4.19$ & $0.16 \pm 0.09$ & $0.22 \pm 0.13$ & $<0.01^{*}$ \\
\hline & & $D_{\max }$ & $54.16 \pm 7.51$ & $54.78 \pm 7.79$ & $0.62 \pm 0.69$ & $0.88 \pm 0.99$ & $<0.01^{*}$ \\
\hline
\end{tabular}

Values are presented as mean \pm standard deviation.

$C T$, computed tomography; PTV, planning target volume; $D_{95 \% \text { ! }}$ dose covering $95 \%$ of target volume; $D_{\text {mean }}$ mean dose; $D_{5 \%}$, dose covering $5 \%$ of target volume; $D_{\text {max }}$ dose delivered to $2 \%$ of organ volume.

${ }^{*} \mathrm{p}<0.05$, Wilcoxon paired signed-rank test. 
same position on the same day to generate synthetic SCT images. The 3D doses calculated based on the SCT images showed good agreement with those calculated from the PCT images, as assessed by image registration, dosimetric parameters, and gamma analysis.

Because of superiority MR for delineation of prostate and normal organs [9], various methodologies generating sCT from MR for implement MR only simulation, such as bulky anatomical density [21-24], machine learning $[25,26]$ and so on, have been suggested. Usually, process of generating $\mathrm{SCT}$ from MRI required a specialist or specific procedure, however, a commercial MRCAT software, used in this study, could generate sCT using the dedicated MR protocol automatically. Especially, Tyagi et al. [23,24] evaluated dosimetric characteristics and implementation of MR only simulation into a clinical work flow, including feasibility of 2D orthogonal digitally reconstructed radiography generated from $\mathrm{SCT}$ or $3 \mathrm{D}$ sCT for IG-IMRT with standard LINACs (linear accelerators) equipped with $\mathrm{kV}$ imager. A clinical workflow of tomotherapy is constructed with a dedicated treatment planning system, dose calculation, delivery quality assurance, and IGRT with MV conebeam CT. We focused on feasibility of MR based tomotherapy for prostate.

During tomotherapy planning, it is necessary to identify the location of the CT couchtop and to replace this location with the couch model of the tomotherapy machine. Since the couchtop is not readily visible on MR images, we attempted to locate the virtual couches on the MR images using couch markers. Since the presence of the couch can affect dose calculation in IMRT [27], it would be beneficial for the sCT algorithm to automatically identify the couchtop location for other rotating treatments, such as volumetric modulated arc therapy.

The DOA station was the only method by which the dose could be recalculated from the SCT images using the previously optimized plan without any additional modifications. Since the DQA workstation does not permit any modifications to the treatment plan, the density of the rectal balloon outline could not be modified and overridden in dose recalculation using this workstation. A rectal balloon was used in both the CT and MR scans; the balloon showed a mean HU of -143.3 on the PCT images. However, since air in the body outline was not allowed in the MRCAT algorithm and the air of the rectal balloon was assigned a mixture of the $\mathrm{HU}$ values of fat and water, the mean $\mathrm{HU}$ of the rectal area on the SCT images was calculated to be 4.6.

Due to the apparent lack of air in the rectal balloon on the sCT images, somewhat higher doses to target volumes were calculated from the SCT images. Thus, the gamma passing rates using the 1\%/1 mm criteria for the PTV1, PTV2, bladder, and rectum were lower than those for other organs (Fig. 3A). However, the overall gamma analysis results with the $3 \% / 3 \mathrm{~mm}$ and $2 \% / 2 \mathrm{~mm}$ criteria showed excellent agreement between the pCT and sCT dose calculations. If the clinical protocol for prostate tomotherapy is established without using the air-filled rectal balloon or if the density of the rectal balloon outline could be modified, this significant difference in target dose would decrease significantly.

In addition, the mean differences in the HUs of bone (both femoral heads), fat, and muscle were calculated to be $-103.4,-7.9$, and 5.7 , respectively. Although the mean HUs of the target vol-
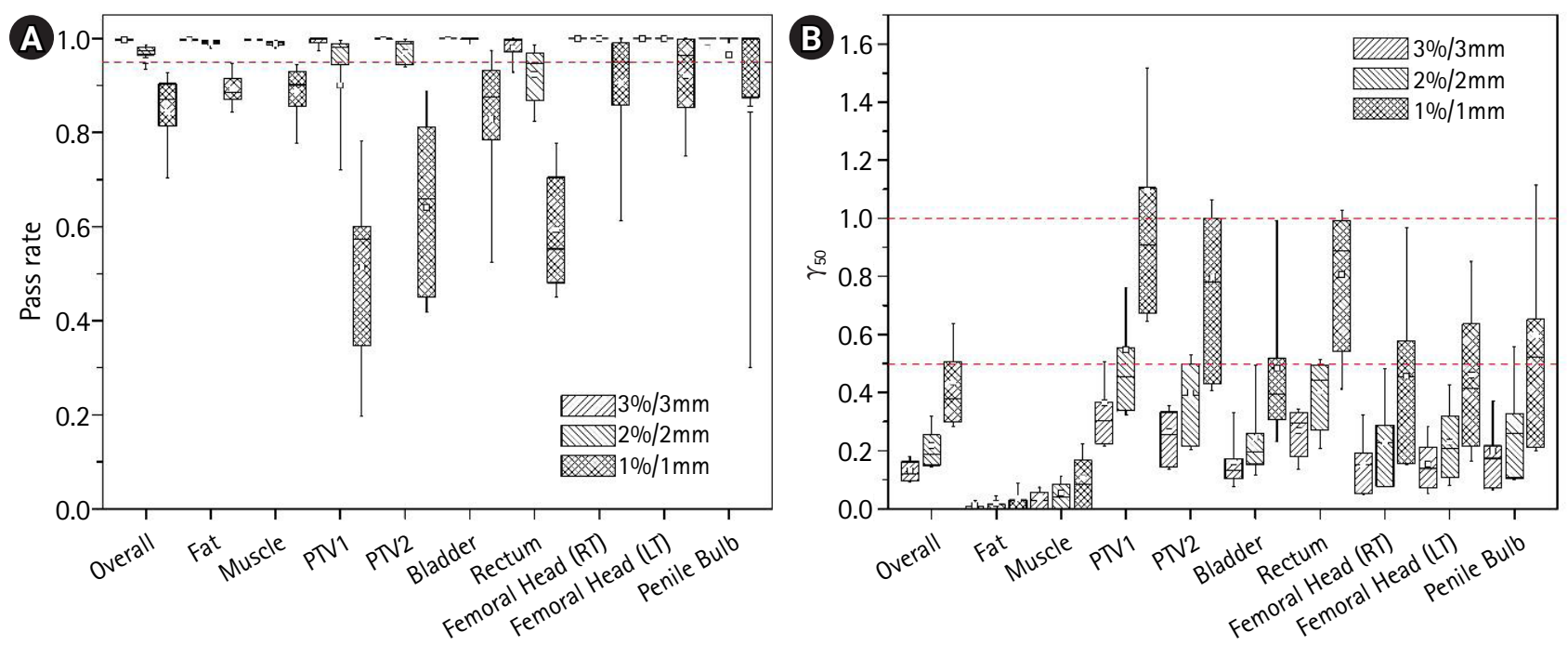

Fig. 3. Gamma analysis results at $3 \% / 3 \mathrm{~mm}, 2 \% / 2 \mathrm{~mm}$, and $1 \% / 1 \mathrm{~mm}$ dose difference/distance to agreement. (A) Pass rates for the target volumes and organs-at-risk and (B) median gamma analysis $\left(\gamma_{50}\right)$ values. PTV, planning target volume; LT, left; RT, right. 
umes and OARs calculated using the SCT images were statistically different from those calculated using the $\mathrm{PCT}$ images, the mean differences were all less than $17 \mathrm{HU}$, with the exception of both femoral heads and the rectal wall. Although these HU differences did not significantly affect the final dose distribution, we intend to improve and reduce these differences in future algorithms. The MRCAT algorithm we used here utilizes five materials (air, fat, normal tissue, spongy bone, and compact bone); we plan to validate other algorithms in future analyses.

We observed small spatial discrepancies in the external body outlines on the SCT and pCT images (Fig. 2C). These discrepancies were due to the use of the anterior coil in the MR scan, which was placed directly on top of the patient. In addition, although we acquired the MR images right after the CT simulation with the patient in the same position, setup uncertainties resulted from the time difference between the CT and the MR acquisitions. However, these uncertainties can be minimized by performing overall dose calculations considering the daily setup variations with fractionated treatments and by using dedicated positioning devices for MR-only simulations [28].

All 16 of our prostate cancer CT simulations and MR simulations were performed on the same day. Although we endeavored to acquire the MR images immediately after the CT simulations, it was sometimes difficult to suitably arrange the logistics of this sequence in busy clinics; moreover, some patients had to wait for their bladders to fill. Despite these limitations, we demonstrated the feasibility of combining tomotherapy with MR-only simulations. We intend to expand this strategy to other radiation therapy techniques using $\mathrm{X}$-ray machines and to other particle therapy machines such as proton and carbon machines.

Inter-target and intra-target delineation variations for prostate cancer with CT-only images can yield variable IMRT planning results. Although rigid or deformable registration using CT and MR provides an avenue by which target structures can be delineated, CT-MR fusion has several limitations such as variable examination time, different immobilization devices, and variations in position. MR-only simulations promise more detailed anatomical information than CT and have the potential to reduce other uncertainties due to their improved soft tissue contrast, single immobilization device setting, and precise treatment positions.

Based on the results of this feasibility study, we anticipate that MR-only simulations will play an important role in IMRT and other advanced treatment techniques for patients with prostate cancer and other types of cancer. Dedicated MR sequences and the MRCAT SCT algorithm were retrospectively applied to determine the radiation therapy workflows. The entire process, including sCT image generation and radiation therapy planning based on SCT, was validated as a feasible approach combining tomotherapy and MR simulation. Tomotherapy planning using MR-only simulation has good accuracy and is clinically feasible with mean dose differences of less than 1\% for the target volumes and normal organs. We intend to implement MR-only simulation tomotherapy in our clinic for treating prostate cancer in order to reduce the time pressure for CT simulation and for other planning and treatment processes. Further studies will focus on improving the MRI scanning protocols, investigating other issues raised in this study, and applying this method to other diseases and treatment machines.

\section{Conflict of Interest}

No potential conflict of interest relevant to this article was reported.

\section{Acknowledgements}

We thank Mark W. Geurts (Department of Radiation Oncology, University of Wisconsin Hospital and Clinics) for conceiving the idea of using a tomotherapy workstation to calculate doses from synthetic CT images. We also thank Teuvo Vaara (Philips Medical Systems MR Technologies, Finland) for helpful discussions of technical issues related to this project.

This study has supported by the National Research Foundation of Korea (NRF) grant funded by the Ministry of Science and ICT (No. NRF-2018R1C1B6006273).

\section{References}

1. Zemplenyi AT, Kalo Z, Kovacs G, et al. Cost-effectiveness analysis of intensity-modulated radiation therapy with normal and hypofractionated schemes for the treatment of localised prostate cancer. Eur J Cancer Care (Engl) 2018;27:e12430.

2. Wortel RC, Incrocci L, Pos FJ, et al. Acute toxicity after image-guided intensity modulated radiation therapy compared to 3D conformal radiation therapy in prostate cancer patients. Int J Radiat Oncol Biol Phys 2015;91:737-44.

3. Pasquier D, Cavillon F, Lacornerie T, Touzeau C, Tresch E, Lartigau E. A dosimetric comparison of tomotherapy and volumetric modulated arc therapy in the treatment of high-risk prostate cancer with pelvic nodal radiation therapy. Int J Radiat Oncol Biol Phys 2013;85:549-54.

4. Rong Y, Tang G, Welsh JS, Mohiuddin MM, Paliwal B, Yu CX. Helical tomotherapy versus single-arc intensity-modulated arc therapy: a collaborative dosimetric comparison between two institutions. Int J Radiat Oncol Biol Phys 2011;81:284-96. 
5. Park JS, Park W, Pyo HR, et al. Suggestion for the prostatic fossa clinical target volume in adjuvant or salvage radiotherapy after a radical prostatectomy. Radiother Oncol 2014;110:240-4.

6. Hentschel B, Oehler W, Strauss D, Ulrich A, Malich A. Definition of the CTV prostate in CT and MRI by using CT-MRI image fusion in IMRT planning for prostate cancer. Strahlenther Onkol 2011; 187:183-90.

7. Smith WL, Lewis C, Bauman G, et al. Prostate volume contouring: a 3D analysis of segmentation using 3DTRUS, CT, and MR. Int J Radiat Oncol Biol Phys 2007;67:1238-47.

8. Park H, Kim JY, Lee BM, et al. A comparison of preplan MRI and preplan CT-based prostate volume with intraoperative ultrasound-based prostate volume in real-time permanent brachytherapy. Radiat Oncol J 2011;29:199-205.

9. Rasch C, Barillot I, Remeijer P, Touw A, van Herk M, Lebesque JV. Definition of the prostate in CT and MRI: a multi-observer study. Int J Radiat Oncol Biol Phys 1999;43:57-66.

10. Buyyounouski MK, Horwitz EM, Price RA, Hanlon AL, Uzzo RG, Pollack A. Intensity-modulated radiotherapy with MRI simulation to reduce doses received by erectile tissue during prostate cancer treatment. Int J Radiat Oncol Biol Phys 2004;58:743-9.

11. Hanvey S, Sadozye AH, McJury M, Glegg M, Foster J. The influence of MRI scan position on image registration accuracy, target delineation and calculated dose in prostatic radiotherapy. $\mathrm{Br} \mathrm{J}$ Radiol 2012;85:e1256.

12. Nyholm T, Nyberg M, Karlsson MG, Karlsson M. Systematisation of spatial uncertainties for comparison between a MR and a CTbased radiotherapy workflow for prostate treatments. Radiat Oncol 2009;4:54.

13. Eilertsen K, Vestad LN, Geier O, Skretting A. A simulation of MRI based dose calculations on the basis of radiotherapy planning CT images. Acta Oncol 2008;47:1294-302.

14. Dowling JA, Sun J, Pichler P, et al. Automatic Substitute computed tomography generation and contouring for magnetic resonance imaging (MRI)-alone external beam radiation therapy from standard MRI sequences. Int J Radiat Oncol Biol Phys 2015; 93:1144-53.

15. Dowling JA, Lambert J, Parker J, et al. An atlas-based electron density mapping method for magnetic resonance imaging (MRI)alone treatment planning and adaptive MRI-based prostate radiation therapy. Int J Radiat Oncol Biol Phys 2012;83:e5-11.

16. Korhonen J, Kapanen M, Keyrilainen J, Seppala T, Tenhunen M. A dual model HU conversion from MRI intensity values within and outside of bone segment for MRI-based radiotherapy treatment planning of prostate cancer. Med Phys 2014;41:011704.

17. Kapanen M, Tenhunen M. T1/T2*-weighted MRI provides clinically relevant pseudo-CT density data for the pelvic bones in $\mathrm{MRI}-$ only based radiotherapy treatment planning. Acta Oncol 2013;52:612-8.

18. Kohler M, Vaara T, Van Grootel M, Hoogeveen $R$, Kemppainen $R$, Renisch S. MR-only simulation for radiotherapy planning. In: White Paper: Philips MRCAT for prostate dose calculations using only MRI data. Amsterdam, the Netherlands: Koninklijke Philips N.V.; 2015.

19. Warner L. MO-D-PinS Room/Hall E-03: Ingenia MR-RT with MRCAT for MR-based radiotherapy simulation. Med Phys 2016; 43(6Part30):3707.

20. Geurts M. TomoTherapy DOA phantom creation [Internet]. San Francisco, CA: GitHub.com; 2015 [cited 2020 Mar 1]. Available from: https://github.com/mwgeurts/tomo_dqa_phantom.

21. Choi JH, Lee D, O'Connor $L$, et al. Bulk anatomical density based dose calculation for patient-specific quality assurance of MRI-only prostate radiotherapy. Front Oncol 2019;0ct 2 9:997.

22. Bratova I, Paluska P, Grepl J, et al. Validation of dose distribution computation on sCT images generated from MRI scans by Philips MRCAT. Rep Pract Oncol Radiother 2019;24:245-50.

23. Tyagi N, Fontenla S, Zhang J, et al. Dosimetric and workflow evaluation of first commercial synthetic CT software for clinical use in pelvis. Phys Med Biol 2017;62:2961-75.

24. Tyagi N, Fontenla S, Zelefsky M, et al. Clinical workflow for MR-only simulation and planning in prostate. Radiat Oncol 2017;12:119.

25. Wang Y, Liu C, Zhang X, Deng W. Synthetic CT generation based on T2 weighted MRI of nasopharyngeal carcinoma (NPC) using a deep convolutional neural network (DCNN). Front Oncol 2019; 9:1333.

26. Liu Y, Lei Y, Wang T, et al. MRI-based treatment planning for liver stereotactic body radiotherapy: validation of a deep learning-based synthetic CT generation method. Br J Radiol 2019;92:20190067.

27. Pulliam KB, Howell RM, Followill D, Luo D, White RA, Kry SF. The clinical impact of the couch top and rails on IMRT and arc therapy. Phys Med Biol 2011;56:7435-47.

28. Sun J, Dowling JA, Pichler $P$, et al. Investigation on the performance of dedicated radiotherapy positioning devices for MR scanning for prostate planning. J Appl Clin Med Phys 2015; 16:4848. 